\title{
Síndrome de Burnout em Médicos Docentes de uma Instituição Pública
}

\author{
The Burnout Syndrome in Teaching Physicians From a Public Service Institution
}

\author{
Vera Lúcia Gama de Mendonça ${ }^{\mathrm{I}}$ \\ Jorge Artur Peçanha de Miranda Coelho \\ Mário Jorge Júca ${ }^{\mathrm{I}}$
}

\section{Resumo}

Trata-se de um estudo correlacional e de comparação entre participantes, em que foram considerados dois conjuntos principais de variáveis: critério (síndrome de burnout e fadiga) e antecedentes (estresse no trabalho e indicadores de situaçáo de trabalho). Participaram deste estudo 60 professores/ médicos de instituiçóes públicas de ensino superior de Maceió - AL, sendo $36(60 \%)$ homens e 24 (40\%) mulheres, com idade variando de 32 até 67 anos $(M=52,31 ; D P=8,41)$. Os participantes foram solicitados a responder a: (a) o Inventário de Burnout de Maslach; (b) a Escala de Avaliaçáo da Fadiga; (c) a Escala de Estresse no Trabalho; além de (d) fornecer suas características sociodemográficas. Evidenciou-se que o estresse no trabalho tem implicaçóes negativas, como a síndrome de burnout e a fadiga. Por fim, chama-se a atenção para necessidade de intervençáo e de mais estudos junto a esta populaçáo.

Palavras-chave: Burnout; estresse ocupacional; fadiga.

\section{Abstract}

This is a correlational and comparative study among participants, in which two main variable groups were considered: criteria (burnout syndrome and fatigue) and antecedents (work stress and work situation indicators). Sixty teaching physicians from higher education public institutions in Maceió, State of Alagoas, Brazil, participated in this study, including $36(60 \%)$ men and 24 (40\%) women, with average age between 32 and 67 years old $(\mathrm{m}=52,21 ; \mathrm{SD}=8,41)$. The participants were requested to answer the Maslach's Burnout Inventory, the Fatigue Evaluation Scale, the Work Stress Scale, and to give their socio-demographic characteristics. It was shown that work stress has negative effects such as burnout syndrome and fatigue, and argued that more studies and interventions in this population are needed.

Keywords: Burnout; occupational stress; fatigue.

Universidade Federal de Alagoas

Os níveis de atenção e concentração exigidos para a realização das tarefas, associados à pressão exercida pelo ambiente de trabalho, são potenciais causadores da síndrome de burnout (ou síndrome do esgotamento profissional, CID-10 Z73.0). Consiste em um tipo de resposta prolongada a estressores emocionais e interpessoais do contexto organizacional. Decerto, "resulta da vivência profissional em um contexto de relaçóes sociais complexas, envolvendo a representação que a pessoa tem de si e dos outros" (Ministério da Saúde do Brasil, 2001, p. 191).

Trigo, Teng e Hallak (2007) argumentam que a prevalência da síndrome de burnout ainda é incerta, mas dados sugerem que acomete um número significativo de indivíduos, variando de aproximadamente 4,0 a $85,7 \%$, conforme a população estudada. "A síndrome afeta principalmente profissionais da área de serviços ou cuidadores, quando em contato direto com os usuários, como os trabalhadores da educação, da saúde, policiais, assistentes sociais, agentes penitenciários, professores" (Ministério da Saúde do Brasil, 2001, p. 192). Não por acaso, alguns estudos têm sido realizados com profissionais de saúde e educação, como por exemplo médicos (Barbosa, Andrade, Carneiro \& Gouveia, 2007), enfermeiros, administradores, nutricionistas, psicólogos, assistentes operacionais e higienistas, no contexto da saúde (Dias, Queirós \& Carlotto, 2010), e professores (Carlotto, 2010; Carlotto \& Câmara, 2007; Gil-Monte, Carlotto \& Câmara, 2010; McCann \& Holt, 2009; Otero-López, Mariño \& Bolaño, 2008; Navarro, Mas \& Jiménez, 2010; Sousa \& Mendonça, 2009; Sousa, Mendonça \& Zanini, 2009).

Destaca-se o recente estudo realizado pelo Conselho Federal de Medicina, sob a coordenação de Barbosa, Andrade, Carneiro e Gouveia (2007), com uma amostra de 7,7 mil médicos de todo o Brasil que reporta dados alarmantes. A maioria deles (57\%) apresenta algum grau preocupante de burnout, sendo que $33,9 \%$ podem ser descritos com manifestação moderada e 23,1\% se enquadram em um nível grave da síndrome. Barbosa et al. (2007) reportam que ao menos um em cada cinco médicos tem vivenciado esgotamento ou estafa resultante do exercício da sua profissão. 
Carlotto (2010) considera que a literatura sobre burnout em professores no Brasil ainda é incipiente, dificultando a comparação com outros estudos nacionais.

Em termos de prevalência, verifica-se que o ensino médio é o nível com maior número de sujeitos acometidos por esta síndrome. Diante desta constatação, e em consonância com os estudos previamente citados, este trabalho aborda o mesmo tema ou correlatos tanto no que diz respeito ao aspecto teórico quanto ao prático, e inventários ou medidas psicométricas utilizadas para aferir estresse no trabalho, burnout e fadiga, como escala de estresse no trabalho (Alves, Chor, Faerstein, Lopes \& Werneck, 2004; Schmidt, Dantas, Marziale \& Laus, 2009); Inventário de Burnout de Maslach (MBI) (Maslach \& Jackson, 1986; Tamayo, 1997; Gouveia, Milfont, Fischer \& Coelho, 2009); e a escala de avaliaçáo da fadiga (Michielsen, De Vries, Van Heck, Van de Vijver \& Sijtsma, 2004; Gouveia, Milfont, Fischer \& Coelho, 2009).

Além disso, trata-se de um estudo correlacional e de comparação entre participantes, com amostra de professores/médicos de instituiçôes públicas de ensino superior de Maceió (AL), do curso de Medicina. Foram considerados dois conjuntos principais de variáveis: critério (síndrome de burnout e fadiga) e antecedentes (estresse no trabalho e indicadores de situação de trabalho, por exemplo: "ocupa ou não cargo de gestão?").

\section{Burnout, Fadiga e Estresse no Trabalho}

Burnout é uma síndrome psicológica em resposta a estressores no trabalho e se constitui de três dimensôes: exaustão emocional, que representa a dimensão básica do estresse individual e refere-se a sentimentos de estar sobrecarregado e esgotado dos próprios recursos emocionais e físicos; despersonalização (ou cinismo), componente representante da dimensáo de contexto interpessoal que diz respeito à resposta negativa, insensibilidade ou resposta de afastamento excessivo dos vários aspectos do trabalho; e realização profissional, descrita como uma tendência do trabalhador se autoavaliar de forma negativa, sentindo-se infeliz e insatisfeito com seu desenvolvimento profissional (Maslach, Schaufeli \& Leiter, 2001; Schaufeli, Leiter \& Maslach, 2009).

Antes de apresentar pesquisas sobre o assunto, é oportuno destacar a diferenciação entre o burnout, entendido como uma resposta ao estresse laboral, de outras formas de resposta ao estresse. Burnout envolve atitudes e condutas negativas com relação aos usuários, aos clientes, à organização e ao trabalho, sendo uma experiência subjetiva que acarreta prejuízos práticos e emocionais para o trabalhador e a organização. Já o quadro tradicional de estresse não envolve tais atitudes e condutas, sendo um esgotamento pessoal que interfere na vida do indivíduo, mas não de modo direto na sua relação com o trabalho (Ministério da Saúde do Brasil, 2001).

Entre os estudos envolvendo esse tema, Carlotto e Câmara (2007) realizaram um trabalho com amostra de 563 professores, sendo 280 de ensino universitário e 283 de ensino não universitário, que exercem atividade docente em instituiçóes particulares na região metropolitana de Porto Alegre (RS). O estudo foi realizado em oito escolas e uma universidade e registrou média (m) de 2,21 e desvio padrão (DP) de 0,67 levando em conta a exaustáo emocional, $\mathrm{m}=1,48$ e $\mathrm{DP}=0,51$ levando em conta a despersonalizaçáo, e $\mathrm{m}=1,53$ e $\mathrm{DP}=0,46$, a realização profissional. Outra pesquisa, realizada por Barbosa et al. (2007) com amostra de 7,7 mil médicos de todo o Brasil, apresenta como resultados para as três dimensóes de burnout: $\mathrm{m}=2,62$ e $\mathrm{DP}=0,78$ para a exaustão emocional; $\mathrm{m}=1,94$ e $\mathrm{DP}=0,70$ para a despersonalização; e $\mathrm{m}=2,03$ e $\mathrm{DP}=0,54$ para a realização profissional.

Quanto à pontuaçáo média nas três dimensôes, fica evidente, para as duas amostras utilizadas nos estudos citados anteriormente, que a exaustáo emocional parece caracterizar o burnout vivenciado pelos médicos $(\mathrm{m}=2,62)$ e professores $(\mathrm{m}=2,21)$, seguida da realização profissional (médicos: $\mathrm{m}=2,03$; professores: $\mathrm{m}=1,53$ ) e, em menor grau, da despersonalização (médicos: $\mathrm{m}=1,94$; professores: $\mathrm{m}=1,48$ ).

Em estudo realizado com uma amostra de 171 professores de Porto Alegre e região metropolitana (34 do ensino infantil, 48 do ensino fundamental, 31 do ensino médio e 58 de ensino universitário), Carlotto (2010) argumenta, com base em evidências, que existe diferença entre os níveis de ensino, sendo a maior prevalência do burnout no nível de ensino médio. A exaustáo emocional parece caracterizar o burnout vivenciado pelos professores de acordo com o nível de ensino, infantil $(\mathrm{m}=1,50, \mathrm{DP}=0,92)$, fundamental $(m=2,57, D P=0,89)$, médio $(m=2,37$, $\mathrm{DP}=0,80)$ e superior $(\mathrm{m}=2,28, \mathrm{DP}=0,66)$; assim como a realização profissional, infantil $(m=4,42$, 
$\mathrm{DP}=0,90)$, fundamental $(\mathrm{m}=3,84, \mathrm{DP}=0,51)$, médio $(\mathrm{m}=2,45, \mathrm{DP}=1,39)$ e superior $(\mathrm{m}=3,11, \mathrm{DP}=0,47)$; e despersonalização, infantil $(\mathrm{m}=0,63, \mathrm{DP}=0,65)$, fundamental $(\mathrm{m}=1,63, \mathrm{DP}=0,81)$, médio $(\mathrm{m}=1,90$, $\mathrm{DP}=0,97)$ e superior $(\mathrm{m}=1,48, \mathrm{DP}=0,45)$. Depreende-se destes resultados que o professor do ensino infantil tenha as melhores condiçóes de trabalho, certamente devido à faixa etária dos estudantes, isto é, "este profissional tem possibilidades de criar ambiente favorável a uma experiência pré-escolar bem-sucedida, não apenas no que se refere à sua interação com os alunos, carregada de afetividade, mas também ao seu papel de profissional e de educador" (Carlotto, 2010, p. 499). Portanto, os menores índices de burnout são apresentados por professores que atuam com crianças quando comparados aos que lidam com níveis de ensino com adolescentes e estudantes universitários.

Além disso, o professor de ensino superior deve conciliar atividades de ensino, pesquisa e extensão, atendendo questóes relacionadas à produção científica, bem como executar atividades administrativas. "Está permanentemente sob um crivo crítico, desde o ingresso na carreira, através de avaliaçôes sistemáticas para a ascensão profissional, da submissão de trabalhos em eventos, da apresentação de projetos e de relatórios de atividades e de pesquisa" (Carlotto, 2003, p. 54). Desta forma, a organização do trabalho do professor tem características que o expóem a fatores estressantes que, se persistentes, podem ter como consequência o burnout e a fadiga.

A fadiga compreende aspectos físicos $\mathrm{e}$ psicológicos, podendo ser entendida como sensação de cansaço físico ou mental, ou sensação de perda de energia ou exaustáo, que resulta na diminuiçáo da capacidade de realizar atividades habituais (Michielsen et al., 2004; Mota, Cruz \& Pimenta, 2005; Pavan et al., 2007). Como reportam Barbosa et al. (2007), aqui também se assume que fadiga é um desconforto generalizado, entendido como sensação subjetiva negativa com componentes comportamental, cognitivo e emocional, isto é, implica em um sentimento de cansaço, desprazer pelas atividades que estão sendo realizadas e relutância em seguir fazendo a tarefa; "é um fenômeno psicofisiológico geral que diminui a habilidade do indivíduo para realizar uma tarefa particular por alternar seu estado de alerta e vigília, bem como sua motivação" (Barbosa et al., 2007, p. 44). Reporta-se que, dependendo dos instrumentos usados e do ponto de corte (cut off) assumido, as taxas de prevalência podem estar no intervalo de 7 a $45 \%$ dos trabalhadores acometidos por esta sintomatologia (Barbosa et al., 2007; Michielsen et al., 2004).

Barbosa et al. (2007) argumentam que a escala de avaliação da fadiga (Michielsen et al., 2004) não apresenta objetivamente um ponto de corte a partir do qual se possa afirmar que a pessoa apresenta este distúrbio. Primeiro, estes autores salientam que se justifica somar todos os dez itens da escala e contar com um indicador unívoco de fadiga, em que as pontuaçôes brutas variam teoricamente de 10 a 50 . Barbosa et al. (2007) apresentam evidências acerca da média deste distúrbio em diferentes amostras de outros países e estabelecem como critério de referência quanto à pontuação total 19 para população geral holandesa, 24 para pacientes croatas com sarcoidose e 29 para pacientes holandeses com sarcoidose. Esses valores de referência foram levado em conta no estudo em questão.

O estresse no trabalho é entendido como uma interação dinâmica entre a pessoa e seu ambiente profissional, com especial atenção para os fatores ambientais e, em particular, os aspectos psicológicos e o contexto organizacional (European Agency for Safety and Health at Work, 2008). Robert Karasek (1979) foi um dos pesquisadores pioneiros a procurar nas relações sociais do ambiente de trabalho fontes geradoras de estresse e suas repercussóes sobre a saúde. Propôs, na década de 1970, um modelo teórico bidimensional que relacionava dois aspectos demandas e controle no trabalho - ao risco de adoecimento (Alves et al., 2004).

As demandas são pressóes de natureza psicológica, sejam elas quantitativas, tais como tempo e velocidade na realização do trabalho, ou qualitativas, como os conflitos entre demandas contraditórias. Já o controle é a possibilidade do trabalhador utilizar suas habilidades intelectuais para a realização de seu trabalho, bem como possuir autoridade suficiente para tomar decisões sobre a forma de realizá-lo (Theorell, 1996, 2000).

Tal como argumenta Alves et al. (2004), o foco do modelo Karasek encontra-se no modo de organização do trabalho. A coexistência de grandes demandas psicológicas com baixo controle sobre o processo de trabalho gera alto desgaste (job strain) no trabalhador, com efeitos nocivos à sua saúde. Também nociva é a situação que conjuga baixas demandas e baixo controle (trabalho passivo), na medida em que podem gerar perda de habilidades e desinteresse. Por outro lado, quando altas demandas e 
alto controle coexistem, os indivíduos experimentam o processo de trabalho de forma ativa; ainda que as demandas sejam excessivas, são menos danosas na medida em que o trabalhador pode escolher como planejar suas horas, de trabalho de acordo com o seu ritmo biológico, e criar estratégias para lidar com as dificuldades (Theorell, 1996). A situação ideal, de baixo desgaste, conjuga baixas demandas e alto controle do processo de trabalho (Alves et al., 2004). Uma terceira dimensão, a do apoio social no ambiente profissional, foi acrescentada ao modelo por Johnson, em 1988 (Theorell, 2000), e definida por seus autores como os níveis de interação social existentes no trabalho, tanto com os colegas quanto com os chefes (Karasek \& Theorell, 1992). Sua escassez também pode gerar consequências negativas à saúde (Alves et al., 2004).

Para a realização deste estudo, o estresse no trabalho foi aferido por meio da escala de estresse no trabalho (job stress scale) adaptada por Alves et al. (2004). Ela é composta por 17 questôes, divididas em três dimensôes: demanda psicológica, controle (discernimento intelectual e autoridade sobre as decisóes) e apoio social.

Alves et al. (2004) realizaram um estudo com esta escala em que se contou com uma amostra de 59 trabalhadores entre enfermeiros, auxiliares e técnicos de enfermagem, escriturários e zeladoras dos períodos diurno, vespertino e noturno. Obteve-se como escore: 12,5 para demanda psicológica, 10,0 para discernimento intelectual, 5,0 para autoridade sobre decisões e 15,0 para apoio social. Note que a dimensão controle está subdividida em discernimento intelectual (dois itens) e autoridade sobre decisóes (também dois itens). Náo são adequados fatores com apenas dois itens; espera-se que o fator esteja constituído de pelo menos três (Pasquali, 2010).

Neste sentido, adotam-se aqui as três dimensôes, sem a subdivisão da dimensão controle. Em consonância com esta decisão, está o estudo realizado por Schmidt et al. (2009). Estes autores levaram em conta uma amostra de 211 trabalhadores de enfermagem de blocos cirúrgicos de 11 hospitais da cidade de Londrina (PR). Reportaram-se os seguintes escores para as três dimensôes da escala de estresse no trabalho: demanda $(\mathrm{m}=14,8, \mathrm{DP}=2,4)$; controle $(\mathrm{m}=16,5, \mathrm{DP}=2,3)$; apoio social $(\mathrm{m}=18,7$, $\mathrm{DP}=2,8)$. Claramente os estudos citados levam em conta as pontuaçóes brutas. Adotou-se o mesmo procedimento neste estudo.

\section{Antecedentes e Consequentes do Estresse no Trabalho}

O estresseé um processo cognitivo, motivacional e relacional. $\mathrm{O}$ termo relacional significa que todas as emoções dependem do que ocorre entre a pessoa e o ambiente, sendo o mais importante as outras pessoas. O indivíduo está constantemente avaliando, isto é, atribuindo significado relacional para continuidade ou mudança de relaçáo com os outros e com o ambiente físico, e é este significado que determina e controla as emoçôes. Se elas são preponderantes para adaptação, então a tradicional divisão tripartida - cognição, motivação e emoção - pode ser incrementada com outro conceito, o de coping. E este está relacionado com os esforços do indivíduo para administrar demandas adaptativas e emoçóes que elas geram (Lazarus, 2006; Segerstrom \& O'Connor, 2012).

Depreende-se que o estresse corresponde às emoçóes negativas que ocorrem toda vez que a pessoa se sente incapaz de lidar com as exigências do ambiente físico e as relações interpessoais, e que é prejudicial para o bem-estar (Lazarus \& Folkman, 1984). Diante desta argumentação, a avaliação cognitiva que o indivíduo faz do significado de um evento, ao invés do evento em si, é que cria as condições da experiência emocional. Aplicando esta noção para a situação ocupacional, o estresse no trabalho é entendido como uma interação dinâmica entre a pessoa e o ambiente, com especial atenção para os aspectos psicológicos e o contexto organizacional. Assim, o estresse é evidenciado a partir da existência de interaçáo problemática pessoa-ambiente ou mensurado em termos de processos cognitivos e reaçóes emocionais subjacentes às interações. Esta conceituação tem sido denominada abordagem psicológica e é notadamente consistente com o conceito de risco psicológico da International Labour Organization e com a definição de bem-estar recomendada pela World Health Organization (European Agency for Safety and Health at Work, 2008).

Parece bastante evidente o papel central das emoçôes no conceito de estresse. Portanto, é necessário, ainda que de forma breve, conceituar e descrever este construto e sua relação com o estresse no trabalho. Emoção é uma combinação de ativação fisiológica, comportamentos expressivos e experiência consciente e desempenha papel central em muitas atividades humanas (Myers, 2009). É um complexo padrão de alteraçóes, incluindo excitação fisiológica, sentimentos, processos cognitivos e 
reaçóes comportamentais, tomadas em reposta a uma situação percebida para ser pessoalmente significativa (Emotion, 2008).

As emoções são multidimensionais e existem como fenômeno subjetivo, biológico, social e com um propósito. São sentimentos subjetivos, pois fazem sentir de determinado modo, que tanto possui significado como importância pessoal. Vale mencionar que os sentimentos são apenas uma parte das emoçôes. Elas também são reaçôes biológicas, ativação biológica ou fisiológica, respostas que preparam o corpo para adaptar-se às situaçóes que o indivíduo enfrenta. As emoções são ainda agentes de um propósito, um estado motivacional (busca de metas) para a execução da ação necessária ao manejo das circunstâncias enfrentadas. Por fim, são fenômenos sociais, dado o seu componente socialexpressivo de comunicação por meio de posturas, gestos, vocalizaçôes e expressões faciais; as emoçôes particulares e subjetivas tornam-se expressóes públicas. Portanto, elas são sistemas sincronizados que coordenam sentimento, ativação, propósito e expressão, de modo a preparar o indivíduo para que se adapte com êxito às circunstâncias da vida (Reeve, 2006; Ekman, 2011). Além disso, são reações aos fatores de estresse ou estressores, ou seja, condições e eventos que evocam tensão ou exaustão emocional. Podem ser eventos simples da vida ou críticos e traumáticos, chegando a se tornarem crônicos.

Sonnentag \& Frese (2003) reportam que os estressores no ambiente de trabalho podem ser agrupados em oito categorias: físicos - condiçóes de trabalho aversivas, tal como barulho, sujeira, elevada temperatura; relacionado à tarefa do trabalho - inclui pressáo por tempo e metas, sobrecarga; função estressora - ambígua ou de conflito, mal definida; social - inclui pobre relação social com chefia, colegas de trabalho, relaçóes interpessoais conflituosas; relacionado ao plano de trabalho - decorrente do tempo de trabalho (longas horas); relacionado à carreira - inclui instabilidade no trabalho e pobre possibilidade de oportunidades; traumáticos - exposição a desastres, acidentes ou atividades de alto risco; mudança organizacional contempla fusão entre organizaçôes, achatamento da estrutura organizacional e implementação de novas tecnologias. As categorias ainda carecem de explícita fundamentação teórica (Sonnentag \& Frese, 2003, p. 455). Mesmo assim, essa taxonomia permite maior clareza quanto ao entendimento das reaçóes ao estresse que afeta o indivíduo fisiologicamente, emocionalmente e em seu comportamento, bem como em seu tempo para atividades de lazer e vida familiar.

As emoçóes negativas (raiva, tristeza e medo, por exemplo) desencadeiam resposta fisiológica ao estresse, o que implica em maior excreçáo de hormônios, como catecolaminas (adrenalina e noradrenalina) e corticosteroides (cortisol) (Sonnentag $\&$ Frese, 2003). Em resumo, o corpo reage ao estresse por meio dos sinais do hipotálamo que chegam às glândulas adrenais, então, adrenalina e noradrenalina são excretadas, elevando a frequência cardíaca e a pressão sanguínea, e ativam o sistema imunológico, reaçoóes necessárias à satisfação das exigências do agente estressor (físicos ou psicológicos). Em seguida, o hipotálamo libera o hormônio liberador de corticotrofina $(\mathrm{CRH})$, e a hipófise produz o hormônio adrenocorticotrófico (ACTH) que ativa o córtex adrenérgico, ocasionando a produçáo de corticosteroides (CO) em humanos, essencialmente o cortisol (Al'Absi, Hugdahl \& Lovallo, 2002; Cohen, Kessler \& Underwook-Gordon, 1997; Lundberg, 2005; Pinel, 2005; Segerstrom \& O'Connor, 2012; Soares \& Alves, 2006). Se o estressor for suficientemente severo, e a exposição do indivíduo a ele prolongada, tem-se como consequência a depleção das defesas somáticas, sendo a resposta ao estresse contínua, e, logo, a vulnerabilidade a desenvolver doenças mentais e físicas.

Entretanto, as respostas individuais são diferentes aos estressores, como argumentam Lazarus (2006) e Segerstrom e O’Connor (2012), seja pelo fator personalidade, pelo manejo do estresse por meio das estratégias de coping, ou ambos. Em síntese, o estresse severo (quanto mais exceder a capacidade do indivíduo de lidar com o estressor e o grau de emoçóes negativas relacionadas a ele) e duradouro for (mais tempo, como uma prolongada e diária jornada de trabalho), maior o impacto somático no indivíduo.

Estudos evidenciam que se o estresse no trabalho é contínuo e intenso, terá como consequência algumas enfermidades, por exemplo: doenças cardiovasculares (Brotman, Golden \& Wittstein, 2007; Habra, Linden, Anderson \& Weinberg, 2003; Lundberg, 2005), problemas imunológicos (Segerstrom \& Miller, 2004) e problemas de saúde mental, como ansiedade, depressão (Burke, Davis, Otte \& Mohr, 2005; Gotlib, Joormann, Minor \& Hallmayer, 2008), falhas de memória (Al'Absi et al., 2002), fadiga (Gouveia et al., 
2009; Michielsen et al., 2004; Mota, Cruz \& Pimenta, 2005; Pavan et al., 2007) e síndrome de burnout (Maslach et al., 2001; Schaufeli et al., 2009), sendo a última o foco principal deste estudo.

\section{Método}

\section{Delineamento}

Trata-se de um estudo correlacional e de comparação entre participantes, em que foram considerados dois conjuntos principais de variáveis: critério (síndrome de burnout) e antecedentes (estresse no trabalho e fadiga).

\section{Participantes}

Contou-se com uma amostra de 60 professores/ médicos de instituições públicas de ensino superior de Maceió (AL), sendo 36 (60\%) homens e 24 (40\%) mulheres, com idade variando de 32 a 67 anos $(\mathrm{m}=52,31 ; \mathrm{DP}=8,41)$. A maioria é casada $(63,3 \%$; $f=38)$, com carga horária de trabalho variando de 20 a 65 horas $(\mathrm{m}=45,8$; $\mathrm{DP}=11,67)$.

A amostra é não probabilística, isto é, de conveniência, tendo a participação de médicos/ professores que, convidados, aceitaram colaborar voluntariamente.

\section{Instrumentos}

Os respondentes receberam um livreto com os seguintes instrumentos psicométricos: MBI, Escala de avaliação da fadiga, Escala de estresse no trabalho (job stress scale) e características sociodemográficas. O primeiro deles foi desenvolvido por Maslach e Jackson (1986) e é composto por 22 itens que englobam três dimensôes: exaustão emocional (9 critérios), realização pessoal no trabalho (8) e despersonalização (5). Foi traduzido e adaptado para o português por Tamayo (1997). No presente estudo foram considerados apenas cinco itens para cada dimensão, tal como reportado no estudo realizado por Gouveia et al. (2009). Os participantes indicaram suas respostas de acordo com uma escala de 1, que representou nunca, a 5, correspondente a sempre. De acordo com Maslach e Jackson (1986), há três níveis de burnout para cada uma de suas três dimensôes: baixo (abaixo do percentil 25), médio (entre 25 e 75) e alto (maior que 75). Carlotto e Câmara (2004) reportam que a versão brasileira do $\mathrm{MBI}$ apresenta parâmetros psicométricos adequados, quanto à validade fatorial e consistência interna, para uso na avaliação de burnout em professores no contexto brasileiro.

A Escala de avaliação da fadiga foi desenvolvida por Michielsen et al. (2004) e compreende dez itens. Para este estudo foi utilizada a versão reportada por Gouveia et al. (2009). Os respondentes indicaram suas respostas numa escala com variação de 1 , significando nunca, e 5, sempre. Como já dito, usaram-se como norma de interpretação os valores reportados por Barbosa et al. (2007) quanto à pontuação total: 19 para população geral, 24 para pacientes croatas com sarcoidose e 29 para pacientes holandeses com sarcoidose. Estes autores indicam que a escala de avaliação da fadiga apresenta parâmetros psicométricos adequados e recomendam o seu uso com amostras de brasileiros. Já a Escala de estresse no trabalho (job stress scale) usou a versão reduzida do questionário originalmente elaborado por Karasek (1979), que continha 49 perguntas, elaborado por Töres Theorell em 1988, com 17 questóes, sendo 5 para avaliar demanda, 6 para avaliar controle e outras 6 para apoio social. Utilizou-se a versão traduzida e adaptada para o contexto brasileiro de Alves et al. (2004). Segundo os autores, os resultados encontrados quanto à validade fatorial e consistência interna permitem considerar a existência de equivalência entre as medidas da escala, em sua forma original e adaptada. Para este estudo foram adotadas as três dimensões, sem a subdivisão da dimensão controle. Em consonância a esta decisão está o estudo realizado por Schmidt et al. (2009).

Finalmente, os respondentes foram requeridos a indicar algumas características sociodemográficas, como sexo e idade, bem como relacionadas à situação laboral, por exemplo: "Qual a sua carga horária de trabalho semanal?", "Ocupa cargo de gestão?”.

\section{Procedimentos}

O primeiro passo foi submeter o projeto ao Comitê de Ética em Pesquisa da Universidade Federal de Alagoas para avaliação e, assim, aprovação (012166/2011-85). A aplicaçáo dos instrumentos psicométricos foi realizada individualmente, por entrevistadores previamente treinados. Num primeiro momento, os participantes foram esclarecidos sobre o estudo em questão e acerca do anonimato e do sigilo 
de suas respostas, para, em seguida, serem solicitados a declarar se estavam ou não dispostos a participar deste estudo. Os que indicaram que sim, foram requeridos a assinar o Termo de Consentimento Livre e Esclarecido. Houve garantia do caráter voluntário da participação, bem como do respeito às diretrizes éticas que regem a pesquisa com seres humanos.

\section{Análise de Dados}

Utilizou-se o Statistical Package for the Social Sciences (SPSS 18) para efetuar as análises estatísticas: descritivas (frequência, percentual, média, desviopadrão, erro padrão e intervalo de confiança de 95\%) e de tomada de decisão (teste $t$ de Student) para comparar os escores médios dos construtos aqui considerados em função das atribuições laborais. Foi realizada análise de correlação $r$ de Pearson para verificar o nível de relaçáo entre as variáveis.

\section{Resultados}

A maioria dos participantes ocupa cargo de gestão $(68,3 \%, f=41)$, atuam em mais de uma instituição $(56,7 \%, f=34)$, não orienta estudantes de pós-graduação $(65 \%, f=39)$, não orienta estudantes de iniciaçấo científica $(55 \%, f=33)$ e metade não orienta estudantes de extensão $(50 \%, f=30)$. Além disso, indicaram carga horária de trabalho variando de 20 até 65 horas $(\mathrm{m}=45,8 ; \mathrm{DP}=11,67)$. Também se buscou conhecer as estatísticas descritivas (média, desvio-padrão, erro padrão e intervalo de confiança - IC de 95\%) das variáveis submetidas a testes de hipóteses neste estudo, tal como descritas na Tabela 1.

Os escores apresentados para as três dimensóes da escala de estresse no trabalho são próximos aos reportados por Schmidt et al. (2009), com amostra de 211 trabalhadores de enfermagem de blocos cirúrgicos. Aqui, observou-se que os professores/médicos apresentam em maior grau controle (escore de 19,32), seguido de apoio social (escore de 19,21) e demanda (escore de 13,43). Em comparaçáo, apresentam maior controle sobre suas atividades e contam com maior apoio social. Entretanto, quando se levam em conta os escores reportados por Alves et al. (2004), de uma amostra de 59 trabalhadores, entre enfermeiros, auxiliares e técnicos de enfermagem, escriturários e zeladoras, o nível de demanda apresentado pelos é um pouco maior. Veja que guardadas as proporçóes do grau de demanda de trabalho para cada contexto, pode-se supor que o trabalho do professor/médico envolve grau de moderado a alto de demanda. Mas, se combinado ao fator controle, que foi evidenciado, depreende-se que se demandas e alto controle coexistem, os indivíduos experimentam o processo de trabalho de forma ativa: ainda que as demandas sejam excessivas, são menos danosas, na medida em que o trabalhador pode escolher como planejar suas horas de trabalho de acordo com o seu ritmo biológico e criar estratégias para lidar com suas dificuldades (Theorell, 1996).

Conciliar atividades de ensino, pesquisa e extensão, atendendo questôes relacionadas à produção científica, além de ter que executar atividades administrativas, são fatores desencadeadores de demanda de trabalho. Potencialmente, esta demanda, sem perder de vista outras variáveis que não foram consideradas neste estudo, como as condiçóes de estrutura física para o trabalho, pode contribuir para o desenvolvimento da síndrome de burnout. Como destaca Carlotto (2003), os professores estáo submetidos a um crivo crítico, desde o ingresso na carreira, por meio de avaliaçóes sistemáticas para a ascensão profissional, da produção acadêmica, da apresentação de projetos e de relatórios de atividades e pesquisa. Esta suposição parece evidente quando se leva em conta a relação entre as variáveis consideradas neste estudo.

Tabela 1. Média, desvio padrão, mínimo e máximo, erro padrão e intervalo de confiança de $95 \%$

\begin{tabular}{lccccc}
\hline Variáveis & Média & Desvio padráo & Mínimo-Máximo & Erro padráo & IC95\% \\
\hline Exaustão emocional & 2,16 & 0,77 & $1,0-4,0$ & 0,10 & $1,96-2,36$ \\
Despersonificação & 1,61 & 0,57 & $1,0-3,4$ & 0,07 & $1,46-1,76$ \\
Realizaçáo profissional & 4,13 & 0,62 & $2,4-5,0$ & 0,08 & $3,96-4,29$ \\
Fadiga & 19,65 & 6,80 & $10-36$ & 0,91 & $17,8-21,5$ \\
Demanda & 13,43 & 2,84 & $7-18$ & 0,38 & $12,6-14,2$ \\
Controle & 19,32 & 2,10 & $15-23$ & 0,28 & $18,7-19,9$ \\
Apoio social & 19,21 & 3,60 & $9-24$ & 0,48 & $18,2-20,2$ \\
\hline
\end{tabular}

IC: intervalo de confiança 
Quanto maior a demanda de trabalho, maior a exaustáo emocional $(r=0,50, p \leq 0,001)$ e despersonificação $(r=0,44, p \leq 0,001)$ e menor a realização profissional $(\mathrm{r}=-0,36, \mathrm{p} \leq 0,01)$. Quanto menor o controle $(\mathrm{r}=-0,36, \mathrm{p} \leq 0,01)$ e apoio social $(\mathrm{r}=-0,51$, $\mathrm{p} \leq 0,001)$ maior o grau de exaustáo emocional. Quanto menor o controle $(\mathrm{r}=-0,36, \mathrm{p} \leq 0,01)$ e apoio social $(\mathrm{r}=-0,29$, $\mathrm{p} \leq 0,001)$ maior o grau de despersonificação, na medida em que quanto maior o controle $(\mathrm{r}=0,51, \mathrm{p} \leq 0,001)$ maior a realização profissional.

A demanda de trabalho está relacionada positivamente a fadiga $(r=0,50, p \leq 0,001)$, enquanto o controle $(\mathrm{r}=-0,54, \mathrm{p} \leq 0,001)$ e apoio social $(\mathrm{r}=-0,41$, $\mathrm{p} \leq 0,001)$ são inversamente proporcionais. Ou seja, quanto maior a demanda, maior a fadiga, e quanto maior o controle e apoio social, menor a fadiga. Neste sentido, o estresse no trabalho está relacionado à síndrome de burnout e à fadiga.

Os escores obtidos neste estudo são semelhantes aos encontrados por outros autores, como é o caso de Barbosa et al. (2007), em uma amostra de médicos de todo Brasil, e Carlotto e Câmara (2007) e Carlotto (2010), com amostras de professores universitários de instituiçôes particulares em relação aos fatores do Inventário de Burnout de Maslach. Quanto à pontuação média nas três dimensôes, observou-se que a exaustáo emocional caracteriza o burnout vivenciado pelos professores/médicos $(\mathrm{m}=2,16)$, seguida da despersonalizaçáo $(m=1,61)$ e, em sentido inverso, realização profissional $(\mathrm{m}=4,13)$. Levando em conta as correlações, verifica-se que quanto maior o nível de exaustáo emocional $(\mathrm{r}=0,66, \mathrm{p} \leq 0,001)$ e despersonificação $(r=0,43, p \leq 0,001)$ maior a fadiga. Em contraste, quanto maior a realização profissional $(\mathrm{r}=-0,46, \mathrm{p} \leq 0,001)$ menor a fadiga. Tal como citado anteriormente, Barbosa et al. (2007) reportam evidências deste distúrbio em diferentes amostras, quanto à pontuação total: 19 para população geral holandesa, 24 para pacientes croatas com sarcoidose e 29 para pacientes holandeses com sarcoidose. $\mathrm{O}$ escore de fadiga obtido neste estudo é semelhante ao da população geral holandesa, porém inferior ao que se observou entre pacientes com sarcoidose. Este resultado indica que os professores/médicos apresentam um grau de fadiga que caracteriza as pessoas em geral. Deve-se ponderar que foram considerados referência escores de amostras de outros países. Além disso, os índices de correlação indicam claramente que quanto maior o estresse e burnout, maior a fadiga.
Buscou-se também analisar se existe diferença entre os escores médios (de estresse, burnout e fadiga) em função dos indicadores de atribuiçóes laborativas, por meio de teste $t$ de Student. Ocupar ou não cargo de gestão, orientar ou não alunos de pós e iniciação científica e atuar ou não em mais de uma instituição não implicam em diferenças estatisticamente significativas (com nível de probabilidade associada de $p>0,05)$ nos escores médios para exaustão emocional, despersonificação, realização profissional, fadiga, demanda, controle e apoio social. Entretanto, o mesmo não foi verificado para o fator demanda, da escala de estresse no trabalho. A diferença entre os escores quanto à situação de orientar ou não alunos de extensão em relaçáo ao fator demanda foi de 0,35 (IC95\% 0,05-0,64), com mediano efeito $(\mathrm{d}=0,65)-$ Cohen (1992) recomenda que, para o tamanho do efeito de teste $t, 0,20$ é um efeito pequeno, 0,50 é um efeito médio e 0,80 é um efeito grande. O teste $t$ $[t(55)=2,383, \mathrm{p}=0,021]$ indicou que existe diferença estatisticamente significativa entre orientar ou não alunos de extensão. Neste estudo, os que orientam $(\mathrm{m}=14,40 ; \mathrm{DP}=2,50)$, apresentam maior escore no fator demanda que os que não o faz $(\mathrm{m}=12,64$, $\mathrm{DP}=3,01)$. Depreende-se deste resultado que a orientação de trabalho de extensão demanda maior carga de trabalho.

\section{Discussáo}

Os resultados deste estudo demonstram a relação entre o estresse no trabalho e a síndrome de burnout e fadiga em uma amostra de professores/ médicos de instituiçôes de ensino superior. Não se trata de um estudo de prevalência, mas providencia mais evidências empíricas de que o estresse no trabalho tem implicaçôes negativas, como a síndrome de burnout e fadiga.

Evidenciou-se que a amostra estudada apresenta alto índice de demanda e controle no trabalho. Neste sentido, quando os indivíduos experimentam o processo de trabalho de forma ativa, ainda que as demandas sejam excessivas, são menos danosas, na medida em que o trabalhador pode escolher como planejar suas horas de trabalho de acordo com seu ritmo biológico e criar estratégias para lidar com as dificuldades (Theorell, 1996). Embora menos danosas, as respostas individuais são diferentes aos estressores, como argumentam Lazarus (2006) e Segerstrom e O’Connor (2012), seja pelo fator personalidade, pelo manejo do estresse por 
meio das estratégias de coping, ou ambos. Levando em conta estas ponderaçóes, os escores aqui encontrados, de estresse no trabalho, burnout e fadiga, são coerentes com os achados das pesquisas supracitadas com outras amostras (Alves et al., 2004; Barbosa et al., 2007; Carlotto, 2010; Carlotto \& Câmara, 2007; Schmidt et al., 2009) e chama a atençáo para a necessidade de intervenção e mais estudos junto a esta população. Em pesquisas futuras, faz-se necessário levar em conta o construto coping e sua medida, para se avaliar o seu papel mediador entre o estresse e burnout e fadiga, por exemplo, bem como analisar o papel dos estressores destacados por Sonnentag e Frese (2003). Também é importante mencionar, tal como reporta Soares e Alves (2006), a possibilidade do exame do estresse por meio da medida de cortisol, como indicador mais verossímil e possibilidade de estudos futuros, para relacionar com as medidas psicométricas. Por fim, mesmo tendo em conta algumas limitaçóes, como o tamanho da amostra, este estudo contribui para a maior compreensão do estresse ocupacional, burnout e fadiga em professores/ médicos do ensino superior de instituiçôes públicas de Maceió (AL), seja por sua expressão na realidade brasileira, bem como pelas necessidades de teorização, investigação empírica e intervenção estruturada neste domínio. Dessa forma, espera-se que as implicaçóes decorrentes e sugeridas possam contribuir e alertar para futuras investigaçóes e intervençóes no contexto educativo que se centrem na promoçáo do bem-estar no trabalho do docente da educação superior.

\section{Referências}

Al'Absi, M., Hugdahl, K., \& Lovallo, W. R. (2002). Adrenocortical stress responses and altered working memory performance. Psychophysiology, 39, 95-99.

Alves, M. G. M., Chor D., Faerstein, E., Lopes, C. S., \& Werneck, G. L. (2004). Versão resumida da "job stress scale": adaptação para o português. Revista Saúde Pública, 38, 164-171.

Barbosa, G. A., Andrade, E. O., Carneiro, M. B., \& Gouveia, V. V. (2007). A saúde dos médicos no Brasil. Brasília: Conselho Federal de Medicina.

Brotman, D. J., Golden, S. H., \& Wittstein I. S. (2007). The cardiovascular toll of stress. The Lancet, 370, 1089-1100.

Burke, H. M., Davis, M. C., Otte, C., \& Mohr, D. C. (2005). Depression and cortisol responses to psychological stress: a meta-analysis. Psychoneuroendocrinology, 30, 846-856.
Carlotto, M. S. (2003). Síndrome de Burnout em professores de instituiçóes particulares de ensino. Aletheia, 17-18, 53-61.

Carlotto, M. S. (2010). Síndrome de Burnout: diferenças segundo níveis de ensino. Psico, 41, 495-502.

Carlotto, M. S., \& Câmara, S. G. (2004). Análise fatorial do Malasch Burnout Inventory (MBI) em uma amostra de professores de instituiçóes particulares. Psicologia em Estudo, 9, 499-505.

Carlotto, M. S., \& Câmara, S. G. (2007). Preditores da Síndrome de Burnout em professores. Psicologia Escolar e Educacional, 11, 101-110.

Cohen, J. (1992). A power primer. Psychological Bulletin, 112, 155-159.

Cohen, S., Kessler, R. C., \& Underwook-Gordon, L. (Eds.). (1997). Measuring stress: a guide for health and social scientists. New York: Oxford University Press.

Dias, S., Queirós, C., \& Carlotto, M. S. (2010). Síndrome de burnout e fatores associados em profissionais da área da saúde: um estudo comparativo entre Brasil e Portugal. Aletheia, 32, 4-21.

Ekman, P. (2011). A linguagem das emoçôes: revolucione sua comunicaçáo e seus relacionamentos reconhecendo todas as expressóes das pessoas ao redor. São Paulo: Lua de papel.

Emotion. (2008, November). Acesso em 20 de Novembro de 2008, em http://www. psychologymatters.org/glossary.html

European Agency for Safety and Health at Work (2008). EU action against climate change: adapting to climate change / European Commission, Directorate-Generale for the Environment. Luxembourg: Offce for Official Publications of the European Communities.

Gil-Monte, P. R., Carlotto, M. S., \& Câmara, S. G. (2010). Validação da versão brasileira do "Cuestionario para la evaluación del Síndrome de Quemarse por el trabajo" em professores. Revista Saúde Pública, 44, 140-147.

Gotlib, I. H., Joormann, J., Minor, K. L., \& Hallmayer, J. (2008). HPA-Axis reactivity: a mechanism underlying the associations among 5-HTTLPR, stress, and depression. Biological Psychiatry, 63, 847-851.

Gouveia, V. V., Milfont, T. L., Fischer, R., \& Coelho, J. A. P. M. (2009). Teoria funcionalista dos valores humanos: aplicações para organizações. RAM, Revista de Administração Mackenzie, 10, 34-59. 
Habra, M. E., Linden, W., Anderson, J. C., \& Weinberg, J. (2003). Type D personality is related to cardiovascular and neuroendocrine reactivity to acute stress. Journal of Psychosomatic Research, 55, 235-245.

Karasek, R. A. (1979). Job demands, job decision latitude, and mental strain: implications for job redesign. Administrative Science Quarterly, 24, 285-308.

Karasek, R. A., \& Theorell, T. (1992) Healthy work: stress, productivity, and the reconstruction of working life. New York: Basic Books.

Lazarus, R. S. (2006). Emotions and interpersonal relationships: toward a person-centered conceptualization of emotions and coping. Journal of Personality, 74, 9-46.

Lazarus, R. S., \& Folkman, S. (1984). Stress, appraisal, and coping. New York: Springer.

Lundberg, U. (2005). Stress hormones in health and illness: the roles of work and gender. Psychoneuroendocrinology, 30, 1017-1021.

Maslach, C., \& Jackson, S. E. (1986). Maslach Burnout Inventory. Palo Alto, CA: Consulting Psychologist Press.

Maslach, C., Schaufeli, W. B., \& Leiter, M. P. (2001). Job burnout. Annual Review of Psychology, 52, 397-422.

McCann, J., \& Holt, R. (2009). An exploration of burnout among online university professors. The Journal of Distance Education, 23, 97-110.

Michielsen, H. J., De Vries, J., Van Heck, G. L., Van de Vijver, F. J. R., \& Sijtsma, K. (2004). Examination of the dimensionality of fatigue: the construction of the Fatigue Assessment Scale (FAS). European Journal of Psychological Assessment, 20, 39-48.

Ministério da Saúde do Brasil (2001). Doenças relacionadas ao trabalho: manual de procedimentos para os serviços de saúde. Brasília: Ministério da Saúde do Brasil.

Mota, D. D. C. F., Cruz, D. A. L. M., \& Pimenta, C. A. M. (2005). Fadiga: uma análise do conceito. Acta Paulista de Enfermagem, 18, 285-293.

Myers, D. G. (2009). Exploring social psychology. New York: McGraw-Hill.

Navarro, M. L. A., Mas, M. B., \& Jiménez, A. M. L. (2010). Working conditions, burnout and stress symptoms in university professors: validating a structural model of the mediating effect of perceived personal competence. The Spanish Journal of Psychology, 13, 284-296.
Otero-López, J. M., Mariño, M. J. S., \& Bolaño, C. C. (2008). An integrating approach to the study of burnout in university professors. Psicothema, 20, 766-772.

Pasquali, L. (2010). Instrumentação psicológica: fundamentos e práticas. Porto Alegre: Artes Médicas.

Pavan, K., Schmidt, K., Marangoni, B., Mendes, M. F., Tilbery, C. P., \& Lianza, S. (2007). Esclerose múltipla: adaptação transcultural e validação da escala modificada de impacto de fadiga. Arquivos de Neuro-Psiquiatria, 65, 669-673.

Pinel, J. P. J. (2005). Biopsicologia. Porto Alegre: Artes Médicas.

Reeve, J. M. (2006). Motivação e emoção. Rio de Janeiro: LTC.

Schaufeli, W. B., Leiter, M. P., \& Maslach, C. (2009). Burnout: 35 years of research and practice. Career Development International, 14, 204-220.

Schmidt, D. R. C., Dantas, R. A. S., Marziale, M. H. P., \& Laus, A. M. (2009). Estresse ocupacional entre profissionais de enfermagem do bloco cirúrgico. Text., \& Contexto Enfermagem, 18, 330-337.

Segerstrom, S. C., \& O’Connor, D. B. (2012). Stress, health and illness: four challenges for the future. Psycholog., \& Health, 27, 128-140.

Segerstrom, S. C., \& Miller, G. E. (2004). Psychological stress and the human immune system: a meta-analytic study of 30 years of inquiry. Psychological Bulletin, 130, 601-630.

Soares, A. J. A., \& Alves, M. G. P. (2006). Cortisol como variável em psicologia da saúde. Psicologia, Saúde \& Doenças, 7, 165-177.

Sonnentag, S., \& Frese, M. (2003). Stress in organizations. In W. C. Borman, D. R. Ilgen, \& R. J. Klimoski (Eds.), Comprehensive handbook of psychology, volum 12: Industrial and organizational psychology, (pp. 453-491). Hoboken: Wiley.

Sousa, I. F., \& Mendonça, H. (2009). Burnout em professores universitários: impacto de percepçóes de justiça e comprometimento afetivo. Psicologia: Teoria e Pesquisa, 25, 499-508.

Sousa, I. F., Mendonça, H., \& Zanini, D. S. (2009). Burnout em docentes universitários. Revista Psicologia e Saúde, 1, 1-8.

Tamayo, M. R. (1997). Relação entre a Sindrome de Burnout e os valores organizacionais no pessoal de enfermagem de dois hospitais públicos. Dissertação de Mestrado náo-publicada. Programa de PósGraduação em Psicologia da Universidade de Brasília, Brasília. 
Theorell, T. (1996). The demand-control-support model for studying health in relation to the work environment: an interactive model. In K. Orth-Gómer, \& N. Schneiderman (Eds.), Behavioral medicine approaches to cardiovascular disease prevention, (pp. 69-85). Mahwah, NJ: Lawrence Erlbaum Associates.

Theorell, T. (2000). Working conditions and health. In L. Berkman, \& I. Kawachi (Eds.), Social epidemiology, (pp. 95-118). New York: Oxford University Press.

Trigo, T. R., Teng, C. T., \& Hallak, J. E. C. (2007). Síndrome de burnout ou estafa profissional e os transtornos psiquiátricos. Revista de Psiquiatria Clínica, 34, 223-233.

\section{Endereço para correspondência:}

Vera Lúcia Gama de Mendonça Universidade Federal de Alagoas

Faculdade de Medicina, Campus A. C. Simóes

Avenida Lourival Melo Mota, s/n

CEP 57072-970 - Maceió/AL

E-mail:vl-mendonca@uol.com.br

Recebido em 20/08/2012

Revisto em 01/10/2012

Aceito em 29/10/2012 